This item was submitted to Loughborough's Research Repository by the author.

Items in Figshare are protected by copyright, with all rights reserved, unless otherwise indicated.

\title{
Modelling defect evolution in irradiated graphite
}

PLEASE CITE THE PUBLISHED VERSION

https://doi.org/10.1016/j.carbon.2019.07.092

PUBLISHER

Elsevier

VERSION

AM (Accepted Manuscript)

PUBLISHER STATEMENT

This paper was accepted for publication in the journal Carbon and the definitive published version is available at https://doi.org/10.1016/j.carbon.2019.07.092

\section{LICENCE}

CC BY-NC-ND 4.0

\section{REPOSITORY RECORD}

Zhou, Ying, Kenny Jolley, Rhiannon Phillips, Roger Smith, and Houzheng Wu. 2019. "Modelling Defect Evolution in Irradiated Graphite". figshare. https://hdl.handle.net/2134/9247898.v2. 


\title{
Modelling defect evolution in irradiated graphite
}

\author{
Ying Zhou, Kenny Jolley, and Rhiannon Phillips \\ School of Science, Loughborough University, Loughborough, LE11 3TU, UK \\ Roger Smith* \\ School of Science, Loughborough University, Loughborough, LE11 3TU, UK and \\ Laboratory of Nanoelectronics and Nanophotonics, \\ Tomsk State University, Lenin Ave, 36, Tomsk, 634050, Russia \\ Houzheng $\mathrm{Wu}$ \\ Department of Materials, Loughborough University, Loughborough, LE11 3TU, UK
}

(Dated: August 3, 2019)

\begin{abstract}
The diffusion of point defects after irradiation events in graphite is considered using high temperature molecular dynamics and adaptive kinetic Monte Carlo. The system is modelled with a ReaxFF potential model. It is shown that monovacancies can diffuse both within the graphite layers and also between layers to form stable divacancy and trivacancy structures. Interstitials can also combine, first forming interlayer strings which transform to ring structures. Separated ring structures can also combine to form mobile platelets which can be the seed for new layer formation. When a defective lattice contains a local mixture of vacancies and interstitials, both recombination and larger defect clusters can form. The Dienes defect, cannot easily occur by direct transformation as originally proposed, because of high energy barriers but is shown to occur as an intermediate step in interstitial-defect recombination process. At high temperature the graphite layers bend which has the effect of enhancing defect motion and changing the relative stability of monovacancy structures. The consequences of this are discussed.
\end{abstract}

Keywords: graphite; defects; long-time scale dynamics; reactive force field

*Corresponding author: r.smith@lboro.ac.uk

\section{INTRODUCTION}

Research into radiation effects in graphite has a long history due to the material having been used both as control rods in the earliest nuclear reactors and also both as a moderator and as a structural material (Gilsocarbon) in the Advanced Gas-cooled Reactors (AGRs) 11. Fine structure nuclear graphite is also likely to be used in future Generation-IV technologies 2. Thus an improved understanding of how graphite behaves under irradiation can help in deciding if lifetimes of existing reactors can be extended and also in understanding how new high temperature reactors can best operate.

In a metal, as a radiation cascade develops, a vacancy rich core forms with an excess of interstitials produced in the periphery. The energy barrier for interstitial diffusion is generally smaller than that for vacancies [3], so although there is some recombination of defects over long time scales, interstitials can diffuse to grain boundaries and surfaces while the vacancies accumulate into voids as the radiation dose increases.

In other materials such as spinels 4, subcascades form and void development is supressed because of structural vacancies and the ability of the material to accommodate a large number of antisite defects which maintain the structure of the material. Other defects that are formed can recombine or decay to antisites over time scales longer than the initial ballistic collisional event.
This makes such materials very radiation resistant and they can withstand a higher number of displacements per atom (dpa) than many materials.

The structure and bonding arrangements in graphite suggest collision cascades would develop differently than in metals and oxides and as pointed out in [5], even before atomistic computer simulation techniques were fully developed for modelling radiation events, early researchers predicted the structure of the collision cascades with remarkable accuracy [6]. In graphite energetically displaced carbon atoms can move between layers and lose energy without necessarily displacing atoms within the layers so damage tends to accumulate at the end of wellseparated sub-cascades. Thus it is not necessary to carry out very large scale molecular dynamics (MD) simulations to investigate a radiation event since the distribution and structure of the resulting defects can be determined by studying sub-cascades of lower energy.

Collision cascades are often successfully simulated using MD and whereas there has been much work carried out investigating such cascades in metals and oxides, it is only over recent years that radiation damage in graphite has been more thoroughly investigated. It was the development of bond order potentials in the 1980s [7] that meant that realistic MD computer simulations in covalent materials could be carried out. The first simulations typically contained only a few thousand atoms due to computing constraints and problems such as how sputtering from graphite could occur [8], how individual particle impacts could form bumps on the surface [9] and how cluster impacts could form hexagonal surface waves 
[10] were investigated. Displacement energy thresholds were also calculated [11].

Since cascades in graphite involve particles channelling through layers and thus simulations require system sizes of several tens of thousand of atoms, the early work did not consider the full development of cascades and it has only been fairly recently with the increase in computational power together with the development of a number of different interatomic potential functions, that full collision cascades in graphite have been investigated [5, 12, 13. For example it was estimated in [5] that a 2 million atoms system would be required to contain a 5 keV cascade. Such MD simulation results have also been used to help interpret high resolution electron microscope images [12].

Although using MD to carry out cascade simulations gives an overall view on the distribution of defects after a collisional event, work by Latham et al 14] comparing the lowest energy defect structures as obtained from the empirical potentials AIREBO [15] and EDIP [16] with ab initio calculations using AIMPRO [17, showed discrepancies between the two approaches. Since DFT results are generally regarded as being more accurate than the empirical potentials, a new reactive force field potential, ReaxFF [18, 19] was fitted to the defect structures obtained from AIMPRO as well as energy barriers for defect transitions. The potential models the defect structures quite well but at the expense of the elastic properties of the material with $c_{33}$ and $c_{44}$ being underestimated compared to experiment. Defect structures in graphite and graphene have been studied using tight binding and ab initio techniques by a number of groups over the years 2023 . An excellent review by Banhart et al 21] examines many of the point defects and the energy barriers between the different metastable structures but apart from some tight binding MD calculations 20] there has been little work examining the dynamical evolution of such defects This is probably because many potentials did not give defect structures in agreement with ab initio calculations and ab initio MD would be too expensive.

The ReaxFF potential combined with adaptive kinetic Monte-Carlo (a-KMC) methods [24, 25] or temperature accelerated dynamics can help the understanding of defect recombination and aggregation after irradiation by identifying dynamical mechanisms in which defects formed from collision cascades can evolve over time scales longer than those normally accessible by MD. Such techniques have had success in modelling other materials and identifying for example, defect clusters that can undergo fast diffusion [26. In graphite, pathways for vacancy motion [20 22, 27] and defect recombination have been previously been investigated using ab initio methods, showing that the recombination of Frenkel pairs can release up to $8.9 \mathrm{eV}$ of energy [27] and a new model ('Ruck and Tuck') 28, was proposed which could further account for stored energy after irradiation. In this model two basal edge dislocations of opposite sign move towards each other and interact to form a folded graphene layer which causes the expansion. The evidence for this mechanism came from high resolution electron microscopy images 29] which showed considerable buckling of the graphitic layers after neutron irradiation. However the way in which irradiation might lead to the formation of such basal edge dislocations remains unclear and buckling can also occur as a result of the accumulation of point defects so further clarification is required.

The paper is laid out as follows. First the methodology is described, followed by a discussion of isolated point defects. Then some MD cascades are carried out to generate typical point defects whose motion over longer time scales is then determined by high temperature MD and a-KMC. Finally the implications of the finding are discussed in terms of the neutron dose effects, lattice structural changes and the Wigner effect.

\section{METHODOLOGY}

The reactive force field (ReaxFF) model for carbon 19. whose repulsive part was splined to a screened Coulomb potential [30] for close interatomic separation, was used to model the interaction between the particles. MD simulations were carried out using the LAMMPS 31 code. An isothermal-isobaric (NPT) ensemble with a Nosé-Hoover thermostat with a variable time step was used for all MD simulations. A conjugate gradient (CG) method was used to relax the system.. Defect motion was analysed in three different ways : (1) by running MD simulations at high temperature, typically $2000 \mathrm{~K}$; This temperature was chosen because it was high enough to see transitions over MD time scales but low enough that it did not cause breaking of bonds or melting. (2) By using the nudged elastic band method (NEB) 32 to determine energy barriers between different known defect states; (3) by using an adaptive kinetic Monte Carlo (aKMC) 25] technique developed at Loughborough which allows open-ended saddle point searches to be carried out over a number of steps where the end state is not known a priori. One simulation was also carried out using PARSPLICE [33] to determine transition times.

Once the energy barriers are determined, the transition rates would normally be calculated using harmonic transition state theory (hTST) by the Arrhenius equation:

$$
r=\tau \exp \left(-\triangle E / \kappa_{B} T\right)
$$

where $\triangle E$ is the activation energy, $\kappa_{B}$ is Boltzmann's constant, $T$ is the temperature and $\tau$ is the prefactor. The NEB calculations used to determine the energy barriers for many of the defects described below, maintain the two dimensional nature of the graphite layers with very little layer distortion. However it will later be shown that because the graphite layers bend at high temperature, transitions can occur much more frequently than what would be expected purely from a consideration of 
energy barriers alone. As a result all transition times that are given later in the paper are calculated by using $\mathrm{MD}$ and not from equation 1 .

\section{RESULTS}

\subsection{Isolated Point Defects}

The ab initio calculations predict a number of metastable interstitial defects [14] and the ReaxFF potential matched their formation energies quite well. Figure 1 shows the most common structures. The spiro Fig. 1 (a) is the most stable structure both in ReaxFF and using AIMPRO [14, 19. AIMPRO gives the next most stable interstitial as the split interstitial with the grafted being the least stable. In 21] for bi-layer graphene, the grafted is more stable than the split interstitial. In this case there is no concept of the split interstitial in the $\alpha$ or $\beta$ site. For the graphite case, there is only a small energy difference between the split interstitial in the two sites.

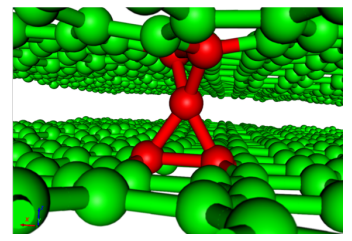

(a) Spiro

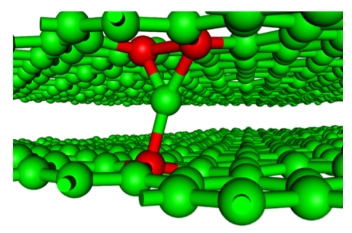

(c) Y-lid

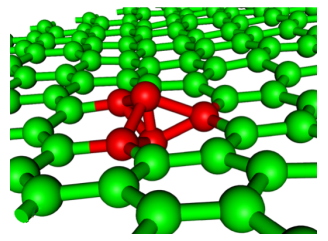

(b) $\beta$-split

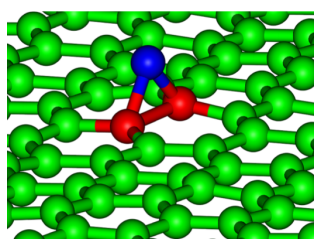

(d) grafted interstitial
FIG. 1: Four common interstitial structures in graphite predicted by ReaxFF potential in agreement with the AIMPRO calculations. Atoms are coloured by coordination number; blue means bonded to two atoms, green 3 and red 4 . The labelling of the interstitial is the same as that used in [14]

The monovacancy structure in graphite is especially difficult to capture exactly with an empirical potential. Fig. 2 visualises what appears to be three geometrically different structures in the way in which the atoms are coloured. The colour scheme used here is the same as in Fig. 1. However it can be seen that small changes in the position of atoms close to the blue atom in Fig. 2 (b) leads to the other two images (a) and (c). In fact with the AIREBO potential all structures have approximately the same energy with an energy barrier for diffusion of $0.11 \mathrm{eV}$, much lower than in the AIMPRO calculations. The ReaxFF potential predicts 3 metastable vacancy structures shown in Fig. 2, ReaxFF gives the structure in (a) as the most stable, $0.6 \mathrm{eV}$ lower in energy than (b) which is in turn lower in energy than (c) by 0.5 eV. DFT calculations with AIMPRO give structure (b) as the most stable with structure (a) a saddle point occurring as structure (a) diffuses. The ReaxFF potential was not fit to structure (a) but as will be shown below, in simulations of vacancy diffusion, we see structures (a), (b) and (c) occurring. In fact although structures (b) and (c) look visually different, the positions of the atoms differ by fractions of an $\AA$.

The energy barriers for monovacancy diffusion using ReaxFF vary between 0.47 from the site shown in Fig, 2 (a) to and adjacent similar site and $0.76 \mathrm{eV}$ from the site shown in Fig2(b) to an adjacent site as shown in Fig.2(a). Based on the equilibrium structures alone it might be expected that the vacancy diffusion pathway in ReaxFF would be from (a) to the adjacent (a) position. It turns out from high temperature MD simulations that this is not the case and that a slight bending of the layers changes the relative stability of structures (a) and (b).

To illustrate this point further, we carried out calculations of the relative stability of the 3 candidate vacancy structures on curved surfaces. In the first example a vacancy was introduced to the middle layer of a triple walled-nanotube as shown in Fig. 3. In this case structure 2(b) was the most stable being $0.2 \mathrm{eV}$ lower in energy than 2(a) and $3.2 \mathrm{eV}$ lower in energy than 2(c). 2(a) occurred as an intermediate state in the diffusion of the vacancy on the surface of the nanotube but the energy barrier for diffusion increased to $1.3 \mathrm{eV}$, about double that for the flat graphite sheet. A further calculation was made in a system when 4 layers of graphite (with periodic boundary conditions) were uniformly bent and when a single bent layer was placed between undistorted layers. The cross-over between the vacancy type shown in Fig. 2(b) being more stable than that shown in Fig. 2 (a) was when the radius of curvature was less than $8 \mathrm{~nm}$ in the uniformly bent case and $14 \mathrm{~nm}$ for the single bent layer.

Since in practice, in a reactor situation, diffusion always occurs at a high temperature, we conclude that when analysing monovacancy motion, the time scale for diffusion is more important than the relative stability of the various candidate structures in the flat undistorted graphitic layer and for divacancy and tri-vacancy structures, the ab initio and empirical potential results are in agreement.

\subsection{Cascades}

A typical collision cascade generated by a $250 \mathrm{eV}$ Primary Knock-on Atom (PKA) in a graphite lattice containing 110,000 atoms is shown in Fig. 4. The cascade is similar in form to those described in [5, 6, 12, 13. The defects after $10 \mathrm{ps}$ of simulation time are relaxed using the conjugate gradient method to the nearest local min- 


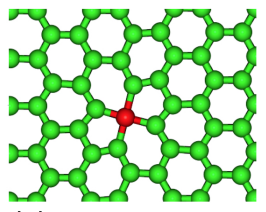

(a)

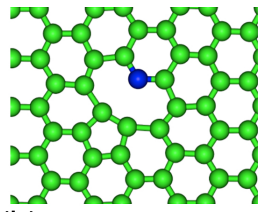

(b)

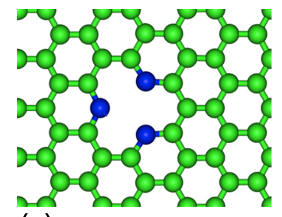

(c)
FIG. 2: Three different vacancy structures in graphite predicted by the ReaxFF potential. Atoms are coloured

by coordination number. (a) split vacancy; (b) $\alpha$ vacancy; (c) vacancy from removal of one carbon atom.

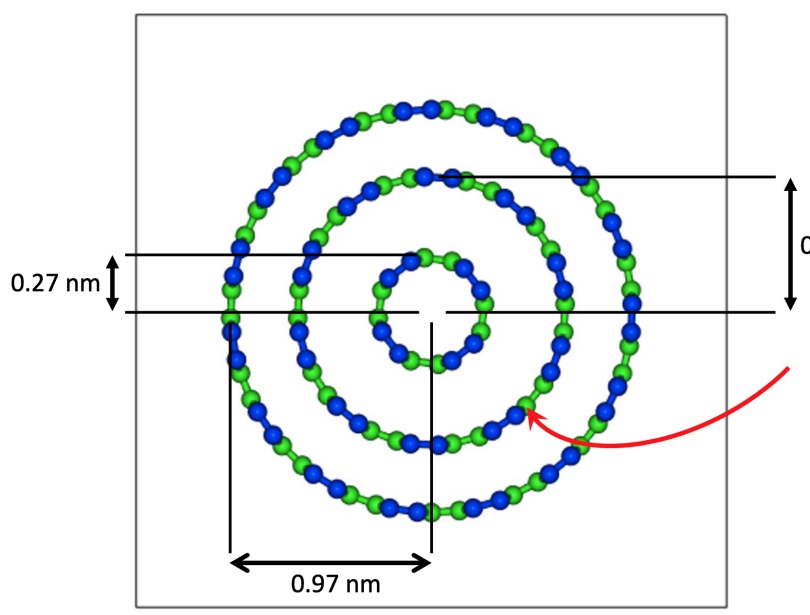

FIG. 3: Vacancy introduced into a triple walled nanotube. The dimension of the box is $3 \mathrm{~nm}$. The red arrow marks the position of the vacancy which is located half way along a nanotube of length $2.3 \mathrm{~nm}$.

imum and are classified in the image. The point defects can be identified as belonging to those that are described in the introduction. Other cascades such as that shown in Fig. 5 at this low energy show similar structures but with metastable interstitial defects not so easily classified as those in the image below. In all cases the metastable interstitial defects produced in the other cascades would relax to the spiro interstitial, or if close to a vacancy recombine with the nearby vacancy over time scales longer than the $10 \mathrm{ps}$ of the MD run. This recombination process is explored later.

The structure of a single cascade is such that generally point defects are produced with the vacancies and corresponding interstitial well-separated. If the point defects are mobile recombination can take place but if the radiation dose rates are high enough the defects can accumulate before recombination can take place.

The metastable interstitials that remain after the cascade shown in Fig 5 were further analysed in order to determine the energy barriers for transformation to the known interstitials shown in Fig 1. The results using the nudged elastic band (NEB) method are shown in Fig. 6 . The energy barrier is around $0.6 \mathrm{eV}$ but the spiro inter-

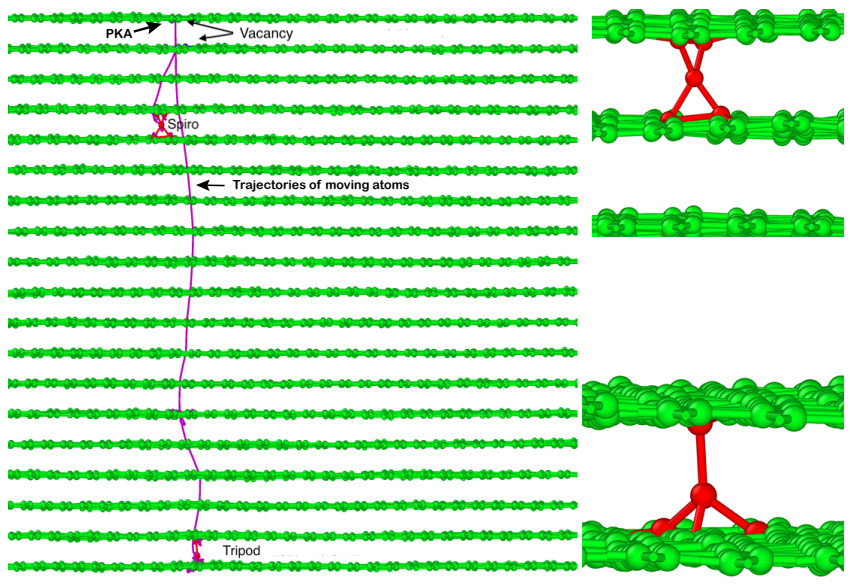

FIG. 4: A trajectory from a $250 \mathrm{eV}$ PKA in a system containing 110,000 atoms at $300 \mathrm{~K}$ showing channelling between the layers. The two vacancies and interstitial defects created by the cascade are clearly marked. The defect structures are shown in more detail on the right hand side of the figure.

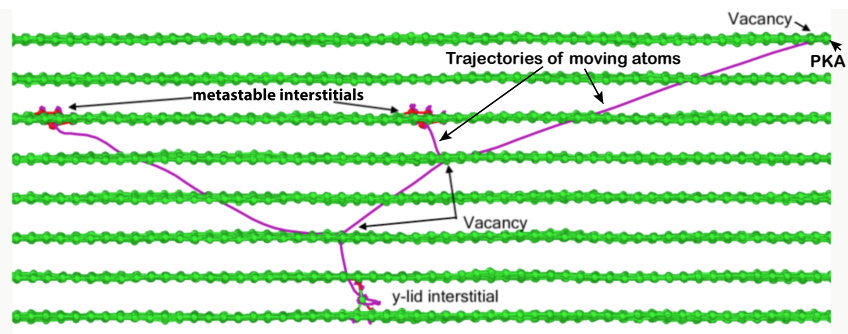

FIG. 5: A trajectory from a $250 \mathrm{eV}$ PKA in a system containing 110,000 atoms at 300K, 6 ps after the initial event. In this case there are 3 Frenkel pairs with two metastable interstitial structures.

stitial is about $1.7 \mathrm{eV}$ more favourable.

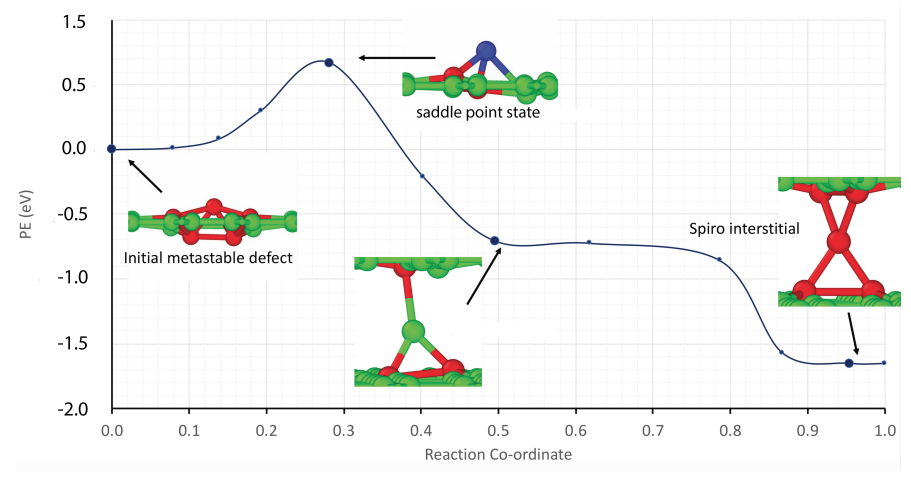

FIG. 6: The transformation of the metastable interstitials that remain 10 ps after a $250 \mathrm{eV}$ collision cascade in Fig 5. The potential energy (PE) shown on the ordinate axis is for the energy relative to the initial state. The line in the inset is a best fir to guide the eye. 


\subsection{Vacancy motion}

To examine how two vacancies in the same graphitic layer interact, two atoms were removed from the layer. The system was allowed to relax and then heated to 2000K to track the defect motion. The system contained initially 800 atoms arranged in 4 layers with periodic boundary conditions. The evolution of the layer containing the vacancies is shown in Fig. 7. The other layers in the system remain intact and are not shown for clarity. After 75 ps the two vacancies join to produce a divacancy 585 ring structure (where the numbers refer to the number of atoms in the rings); this remained stable and did not diffuse further.

It can be seen that at $2000 \mathrm{~K}$ that the layers bend and that atoms are also displaced from the mean position of the layers. Little change in the in-plane bond lengths was observed but there is a variation in the position of the atoms in the $c$ direction. This point is further discussed in the discussion session below.

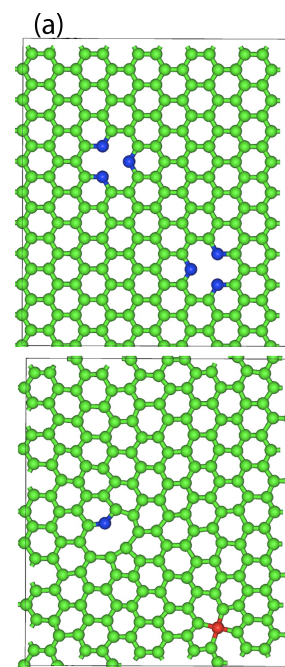

(d)

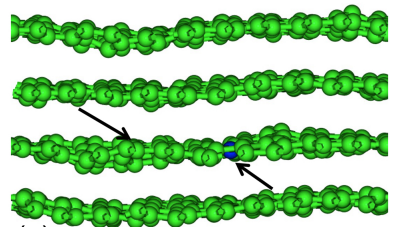

(g) (b)

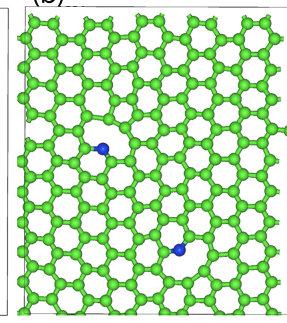

(c)

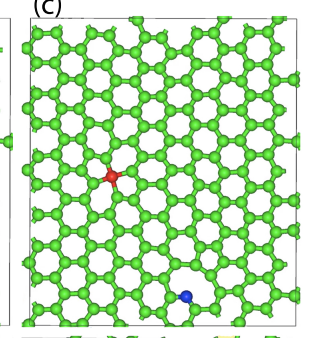

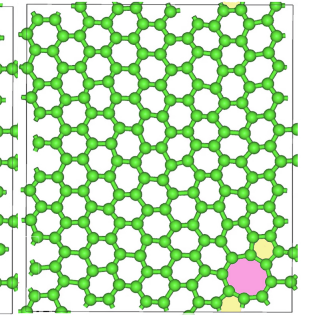

(f)

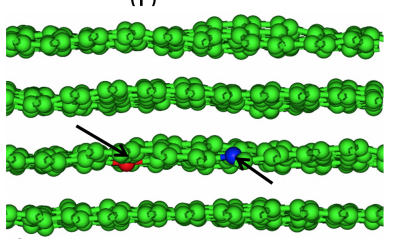

(h)

FIG. 7: Structures with two mono-vacancies evolving at $2000 \mathrm{~K}$ Atoms are coloured by coordination number.

(a) 0 ps with two atoms removed from a perfect graphitic layer; (b) 15ps; (c) 30ps; (d) 40ps; (e) 60ps;

(f) $75 \mathrm{ps}$. This structure is the 585 ring divacancy which is shown shaded for clarity. (g) and (h) correspond to images shown in (c) and (d) illustrating the displacement of atoms in the $c$ direction.

A number of potential structures involving two vacancies are shown in Fig. 8. The di and tri vacancy structures that evolve have been reported in the literature

before for both graphene and graphite [21, 23]. In Fig. 8 (a) there are two $\alpha$-vacancies in a single layer and in Fig. 8 (b) there are two $\alpha$-vacancies in two adjacent layers. Fig. 8 (c) and (d) show a 585 ring and 555777 ring. All these structures were found during high temperature MD simulations. Taking the structure shown in Fig. 8 (a) as the reference, Fig. 8 (b) is higher in energy by $0.68 \mathrm{eV}$ while structure (c) is lower in energy by $8.56 \mathrm{eV}$ and structure (d) by $9.9 \mathrm{eV}$. It also requires $2.5 \mathrm{eV}$ for a 585 ring to transform to a 555777 ring while the reverse barrier is $3.9 \mathrm{eV}$. This suggest that the 585 divacancy will be very stable once it has formed. We did not observe this transformation using MD so simulations using PARSPLICE 33] were undertaken. The transition was observed to occur after 9 ns of simulation time at $2000 \mathrm{~K}$.

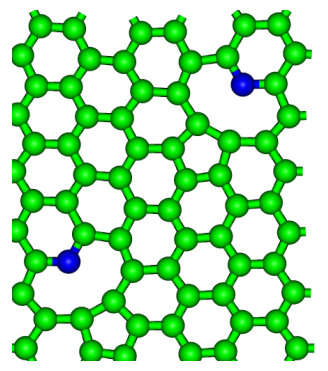

(a)

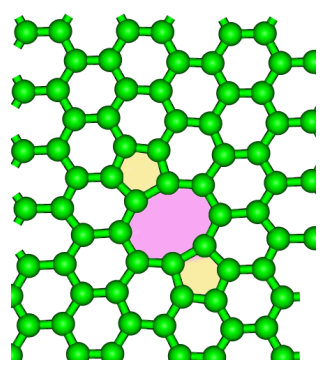

(c)

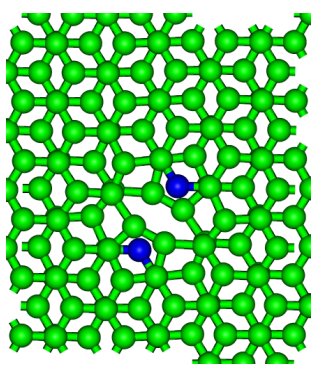

(b)

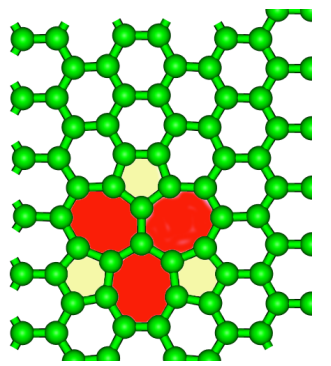

(d)
FIG. 8: (a) Two separated mono-vacancies in the same graphitic layer; (b) Two mono-vacancies on top of each other in adjacent layers; (c) The 585 divacancy structure; (d) The 555777 divacancy structure.

MD simulations were undertaken at $2000 \mathrm{~K}$ when three monovacancies were present in the same graphitic layer. The system contained 4 layers with periodic boundary conditions and 200 atoms in the complete layers. The simulation was initialised by removing 3 separate carbon atoms and allowing the system to evolve. The energy differences between Fig. 9 (a) and (b) is $16.7 \mathrm{eV}$, so Fig. 9 (b) represents a very stable structure which was reached after about 100 ps of simulation time.

a-KMC calculations were also carried out when a system was initialised with two vacancies in adjacent layers. Transitions were only found when the vacancies formed as in Fig. 8(b) which is equivalents to Fig. 10(a). The con- 


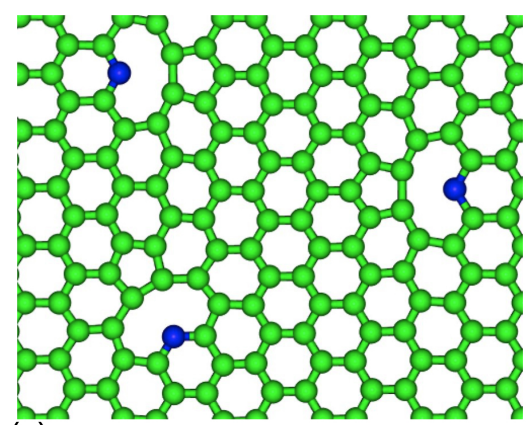

(a)

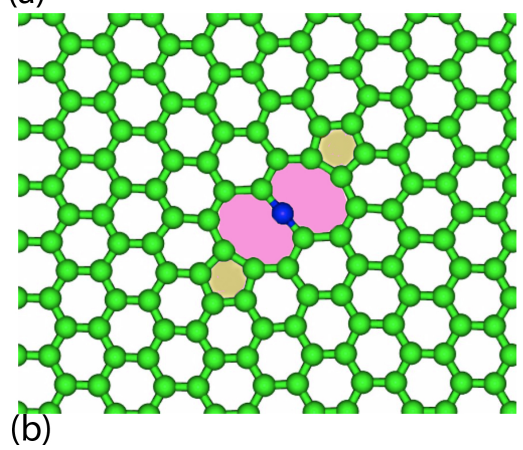

FIG. 9: MD also shows that three separated monovacancies within a layer can also recombine into a tri-vacancy structure. The energy difference between the starting position (a) and the end position (b) is 16.7 $\mathrm{eV}$ i.e. (b) is much more stable.

figuration in Fig. 10 (a) consists of two adjacent monovacancies in different layers. The first transition shown in (b) is where two under co-ordinated atoms from each monovacancy bond to form a chain joining the layers together; after running MD simulations for a $426 \mathrm{ps}$ at $2000 \mathrm{~K}$, one carbon atom in the lower (blue) layer joined the upper layer which became a perfect graphitic sheet leaving a 585 ring in the layer below. This is the transition examined in 27] using AIMPRO. The calculations carried out in 27] indicate that a large barrier of around $7 \mathrm{eV}$ needs to be overcome for this process to occur but that the end structure is about $8 \mathrm{eV}$ lower in energy than the isolated vacancies, a value comparable to the 8.56 of ReaxFF. However in our simulations with ReaxFF when the layers bend, the recombination occurs faster than the $10 \mathrm{eV}$ energy barrier would suggest. The other difference is that our metastable state in Fig. $10 \mathrm{~b}$ is a saddle point in [27.

A further a-KMC calculation on a three-vacancy system showed that multiple vacancies spanning different layers can accumulate into one layer; for example, when two vacancies lie next to each other in adjacent layers and a further monovacancy also exists in one of the layers. In this case the 5885 trivacancy structure, shown in Fig. 9(b) can form after 10 a-KMC steps.

\subsection{Interstitial aggregation}

Table I lists the forward and reverse energy barriers between the interstitial structures predicted by ReaxFF calculated using the NEB method. It can be clearly seen that the spiro interstitial is the most stable and that other interstitial structures formed in a collision cascade would decay to the spiro interstitial over long time scales.

As shown in Fig. 11 it requires $0.98 \mathrm{eV}$ for a spiro interstitial to diffuse within two graphitic sheets and 1.74 $\mathrm{eV}$ between layers. a-KMC shows that the latter process occurs through a replacement mechanism as shown in Fig. 11 (b).

TABLE I: Energy barriers (eV) between structures, with labelling for the interstitials as shown in Fig. 1. F refers to the forward barrier and $\mathrm{B}$ the reverse barrier.

The DFT calculations are : (a) our own values determined with AIMPRO; (b) values from bi-layer graphene [21]; (c) data from [34]; (d) data from [20].

\begin{tabular}{cc|cc|c|c}
\hline & & \multicolumn{2}{|c|}{ ReaxFF } & DFT & \\
\hline Initial & Final & $\mathrm{F}$ & $\mathrm{B}$ & $\mathrm{F}$ & $\mathrm{B}$ \\
\hline \hline Spiro & $\beta$ split & 1.5 & 0.8 & & \\
spiro & Y-lid & 0.9 & 0.7 & & \\
spiro & grafted & 1.5 & 0.4 & $2^{a}, 1.3^{b}$ & $0.3^{a}, 0.8^{b}$ \\
Y-lid & grafted & 1.4 & 0.5 & & \\
spiro & spiro & 1.0 & - & $2.1^{b}$ & - \\
grafted & grafted & 0.6 & - & $0.4^{a}$ & - \\
grafted & $\beta$ split & 0.6 & 1.0 & $0.5^{a}, 0.4^{b}$ & $0.2^{a}, 0.5^{b}$ \\
$\alpha$ split & grafted & 0.8 & 0.4 & $0.7^{a}$ & $0.3^{a}$ \\
vacancy vacancy & $0.5-0.7$ & - & $1.2-1.4^{c}$ & - \\
Dienes & & 8.0 & 3.1 & $9.6^{a}, 9.2^{d}$ & $4.5^{a}, 4^{d}$ \\
\hline
\end{tabular}

When there are multiple interstitials, they can join together and form chains and rings between the graphite layers. Fig. 12 shows that two adjacent spiro interstitials between layers are less favourable than if they are separated or located in adjacent layers. In addition MD simulations show that they easily combine to form a two atom chain between layers as shown in Fig. 12 (d). This configuration is in agreement with ab initio calculations [22, 35] where both this structure and the di-interstitial, grafted onto one of the graphite layers, are the lowest energy structures. However the grafted structure never occurred in dynamical simulations as it would require much more rearrangement to form and as pointed out in 22], ab initio calculations show that there is a large energy barrier of $2.27 \mathrm{eV}$ to be overcome before it can form. Three adjacent spiro interstitials combine to form a 3 atom chain as shown in Fig. 13. The simulations show that when 2 or 3 interstitials are located close to each other they transform to form chains between the layers, so a simulation experiment was undertaken whereby 20 spiro interstitials were randomly placed between two graphitic layers in a system containing 2048 atoms arranged in 4 layers with periodic boundary conditions. The system was then heated to $2000 \mathrm{~K}$ and allowed to 


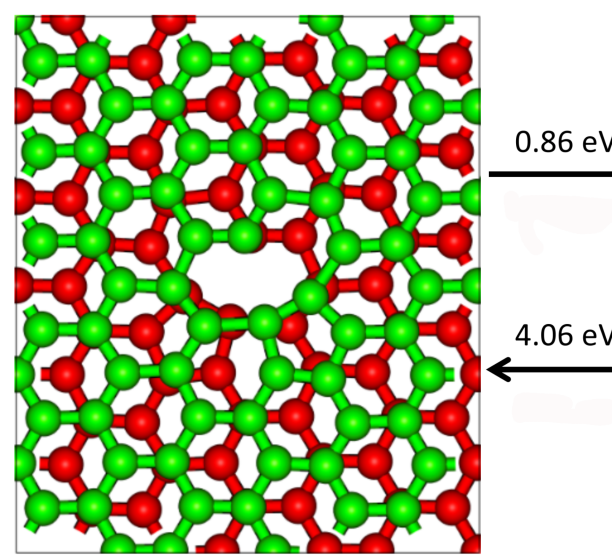

(a)

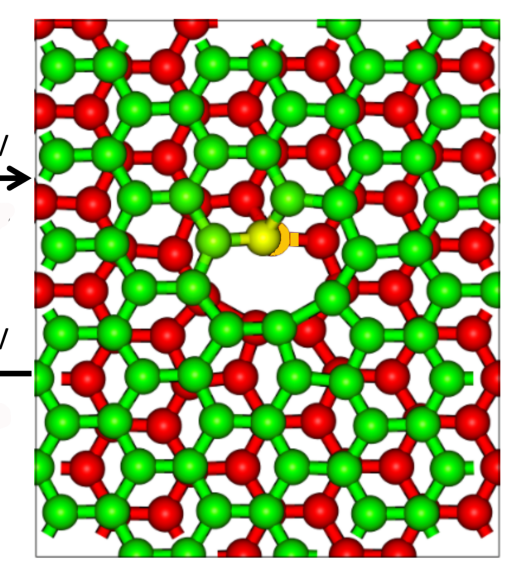

(b)

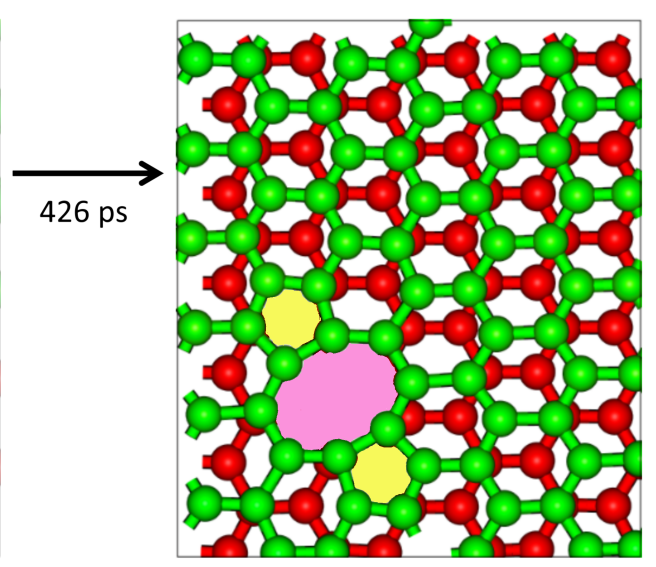

(c)

FIG. 10: The coalescence of two mono-vacancies in adjacent layers, as determined by a-KMC. Atoms are coloured according to which layer they are in, with the yellow and gold colours in (b) representing bonded atoms between the layers. Only two graphitic layers are shown for clarity. The red atoms are in the layer below the green atoms. The

final structure is a 585 divacancy structure which is shown shaded in the diagram for clarity. (a) the starting structure as shown in Fig. 8(b); (b) an intermediate structure where two atoms bond from adjacent layers; (c) the final structure with one complete graphitic layer and a 585 divacancy. The transitions between structures (a) and (b) were determined by a-KMC. The final transition from (b) to (c) was determined from one MD simulation at $2000 \mathrm{~K}$.

evolve using MD.

The starting configuration is shown in Fig. 14(a). After 500 ps some of the interstitials have migrated to lie between different layers and shown in Fig. 14(c). After this time only the motion of the interstitial atoms between the original layers are plotted for clarity. After 575 ps as shown in Fig. 14(d) they form a chain which evolves into a ring structure with a tail after 650 ps and then into a mobile ring structure after 750 ps as shown in Fig. 14(f).

\subsection{Platelet formation and motion}

Since the simulations have shown that spiro interstitials can aggregate into ring structures that are mobile between the layers, a further simulation experiment was carried out by arbitrarily placing 3 artificially generated ring structures between 2 graphitic layers, heating the system again to $2000 \mathrm{~K}$ and tracking the motion using MD. The results are shown in Fig. 15 where (a) is the artificially generated starting structure. After 2 ns the structure has evolved into a single platelet structure consisting of mostly 6 membered rings as shown in Fig. 15(d). This platelet is highly mobile between the graphite layers at $2000 \mathrm{~K}$. The above set of simulations shows how clusters of interstitial atoms can evolve together to form platelets which in turn could grow to form new graphite layers.

\subsection{Vacancy-Interstitial recombination}

In the case where a vacancy and interstitial are located close to each other, recombination can take place in two separate ways. In case 1 , the recombination yields a perfect graphite layer with the highest energy barrier in the recombination process being $0.31 \mathrm{eV}$. MD simulations show this transition occurring after approximately 43 ps at $2000 \mathrm{~K}$. In the second case the resulting structure consists of two seven member and two five membered rings (Dienes defect [36, 37]). This transformation takes around 63 ps at $2000 \mathrm{~K}$. MD shows that both transformations are possible and that the resulting structures are quite stable. The direct transformation from a perfect graphite structure to the 5577 system was originally proposed by Dienes [36] and Thrower 38] as a means by which diffusion in graphite could take place. It is sometimes also called a Stone-Wales 39 transformation. However the direct transformation has an extremely high energy barrier at over $8 \mathrm{eV}$ as calculated using ReaxFF. See Table I for the comparison with DFT. It is thus unlikely to occur as a diffusion mechanism but the simulations here show that it might occur as an intermediate step in an interstitial-vacancy recombination process. The reverse barrier for this defect to revert back to the perfect lattice is also high at $\approx 3 \mathrm{eV}$. However two separate MD simulations were undertaken when a system containing 2000 atoms containing a Dienes defect was heated to $2000 \mathrm{~K}$. In the first case the perfect lattice reconstructed in $78 \mathrm{ps}$ and in the other case $540 \mathrm{ps}$, much faster than would be expected from equation 1 . This suggests that layer bending due to temperature also has the 

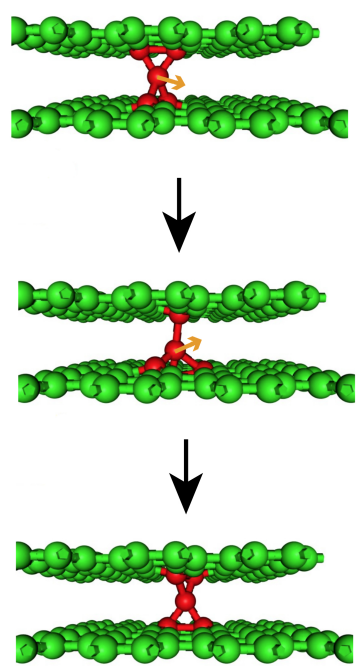

(a)

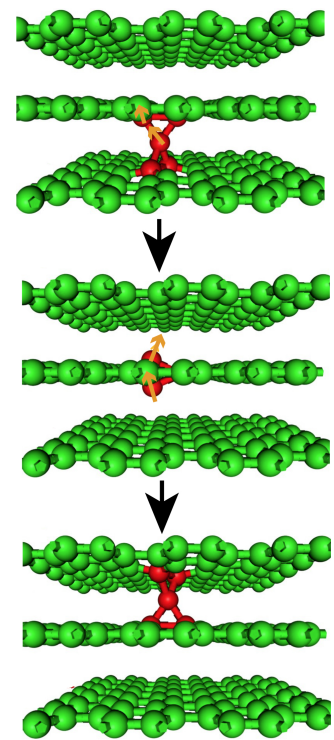

(b)

FIG. 11: The diffusion of spiro interstitial (a) within a layer and (b) between layers. Atoms are coloured by coordination number. The yellow arrows indicate the direction of transitions. The energy barriers and transitions were determined by a-KMC. The middle figure in (a) is the saddle point state and this transition has an energy barrier of $0.98 \mathrm{eV}$. In (b) There is a replacement mechanism with an energy barrier of 1.74 $\mathrm{eV}$ with the split interstitial as an intermediate state. It is also possible for the spiro to rotate but with no net diffusion. The energy barrier for this is $0.5 \mathrm{eV}$.

effect of changing the energy barriers, in this case causing an effective reduction.

Fig. 16 shows an example where 10 vacancies and 5 interstitial are randomly distributed between 2 layers in a system containing 4 graphite layers. The total number of atoms is 4027 and periodic boundary conditions are applied. The system is heated for $2 \mathrm{~ns}$ at $2000 \mathrm{~K}$. Fig. 16(a) shows the starting configuration and (b) the structure after 2 ns. There are 2 spiro interstitials remaining after 2 ns and in layer 2 shown in (b), 2 monovacancies are still present. There is a Dienes defect (left hand red ellipse) and a 585 divacancy structure. In layer 3 there is a tri-vacancy structure. Once the di and tri-vacancy structures have formed they were not seen to diffuse over the 2 ns time scale of the simulation. The monovacancy structures continually diffuse and change shape between the 3 configurations shown in Fig. 2.

\section{DISCUSSION AND CONCLUSIONS}

The defect formation energies predicted by ReaxFF were fitted to, and are in good agreement with, the ab initio results [19, in contrast to some existing potential

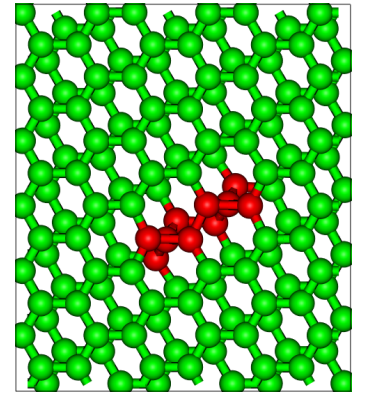

(a)

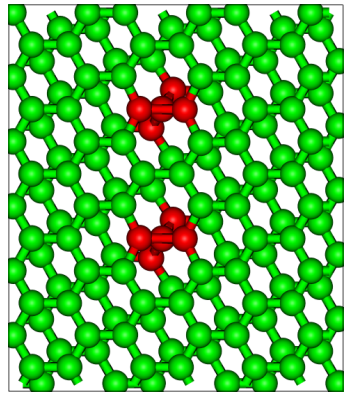

(b)

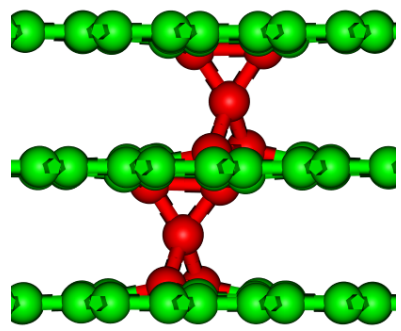

(c)

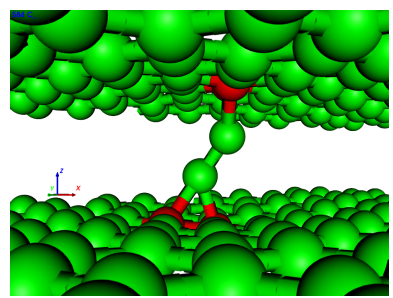

(d)

FIG. 12: Configurations of two interstitials in close proximity. (a) Two adjacent spiro interstitials; (b) Two separated spiro interstitials $(0.16 \mathrm{eV}$ lower in energy than (a); (c) Two spiro interstitials in adjacent layers, $0.35 \mathrm{eV}$ lower in energy than (a); (d) A two-atom chain between two graphitic layers $(2.7 \mathrm{eV}$ lower in energy than (a). Atoms are coloured by coordination number. The perspective in (a) and (b) is slightly offset from the graphite $c$ axis.

models 14 and although monovovacancies differ, the di and tri vacancy structures are in agreement. There are fewer results in the literature on diffusion pathways but the potential model is in reasonable qualitative agreement with the barriers and pathways that are given in the literature.

The simulations have shown how point defects in graphite, formed by irradiation can combine. In the case of isolated vacancies, diffusion can occur both within a layer and between layers to form stable di and tri-vacancy structures. Single vacancy diffusion in a layer has an energy barrier which is lower than the diffusion of the spiro interstitial. Vacancies can only diffuse between layers when situated above a corresponding vacancy in the next layer forming a divacancy in one layer and a com- 


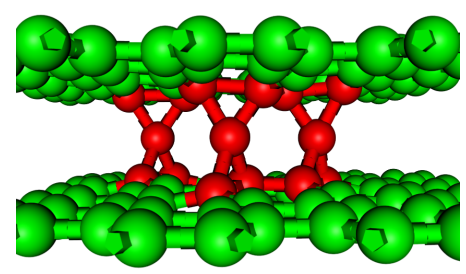

(a)

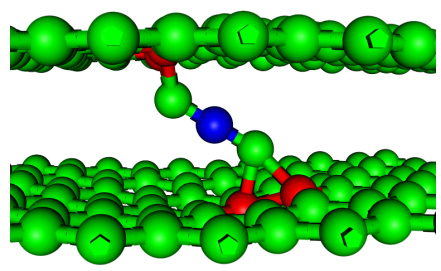

(b)

FIG. 13: Configurations of three spiro interstitials and three-atom chain. Atoms are coloured by coordination number. In (a) three spiro interstitials are located close to each other between the same two graphite layers. a-KMC simulations show a transition from (a) to the structure shown in (b) which is $1.34 \mathrm{eV}$ lower in energy.

Atoms are coloured by co-ordination number.

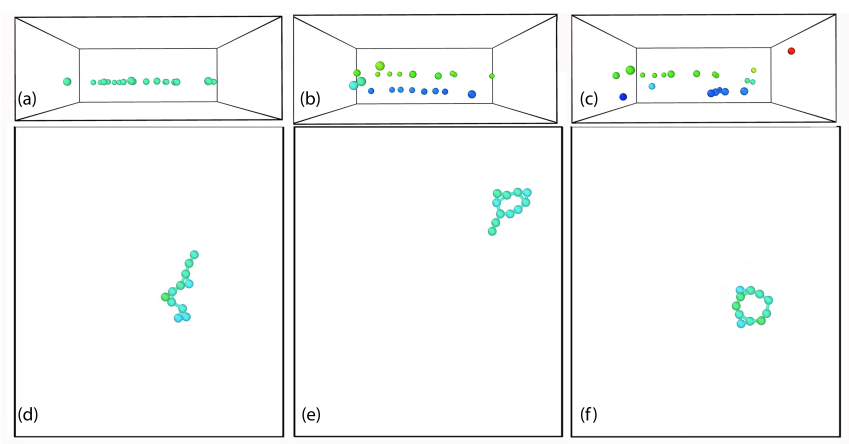

FIG. 14: Evolution of 20 spiro interstitials placed initially close together between two graphitic layers and annealed at $2000 \mathrm{~K}$. (a)-(c) show the evolution as a side view (a) is the starting arrangement; (b) after 250ps;

(c) 500ps; (d)-(f) show only the evolution of those atoms trapped between layers in the middle of the structure. (d) $575 \mathrm{ps}$; (e) $650 \mathrm{ps;} \mathrm{(f)} 725$ ps. Atoms are coloured by height to illustrate the position of the interstitials between different layers.

plete layer in the other. The di and tri-vacancy structures formed by accumulation of vacancies are effectively immobile, even at high temperatures.

When interstitials and vacancies are situated close to each other they can recombine, forming either a Dienes defect or a perfect hexagonal layer, releasing up to $9 \mathrm{eV}$ of energy into the system. The Dienes defect has too high an energy barrier to form by self diffusion but was observed to occur as an intermediate metastable structure in a vacancy-interstitial recombination process.

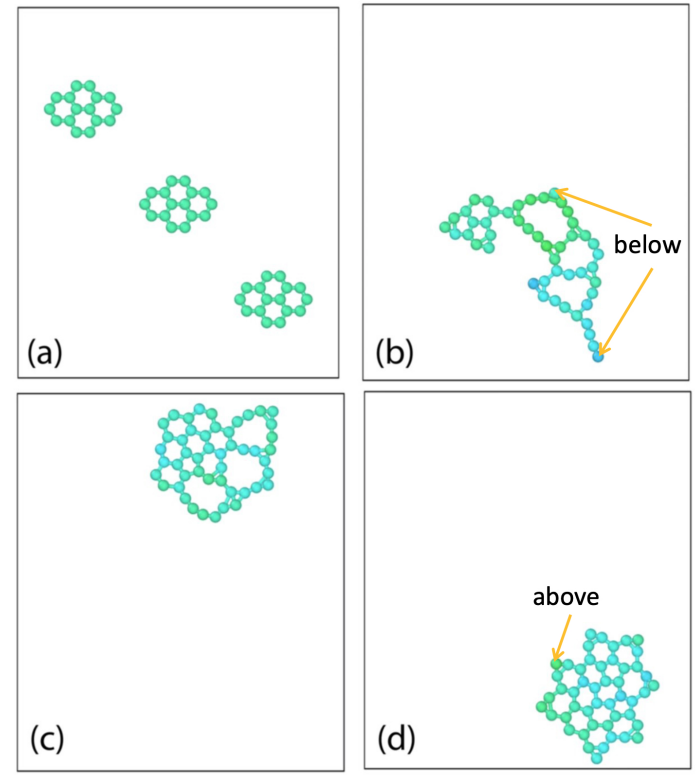

FIG. 15: Evolution of 3 ring structures placed close together between two graphitic layers and annealed at 2000K. The system contain 4 layers of graphite with periodic boundary conditions and 512 atoms in each layer. Only the interstitial atoms are shown for clarity. (a) The artificially generated starting structure; (b) the structure after 50 ps ; (c) 100 ps ; (d) 2 ns. During the high temperature simulations some atoms near the edge of the platelet can bond and debond with the adjacent

layers. Such atoms are labelled in the diagram.

An important observation from these results is that the bending and buckling of the graphite layers has a large effect on the defects both in terms of their relative stability and also in terms of the defect mobility. The transitions times cannot be determined by using harmonic transition state theory. The layers bend at high temperature and defects can diffuse more quickly than would be expected from energy barriers determined from the NEB method for flat graphite and then used in the Arrhenius equation to determine transition times. This effect was observed when determining vacancy diffusion, divacancy transitions and Dienes defect recombination and appears to be a general consequence of the two dimensional nature of graphite. The fact that graphite layers can be subject to wave-like motion has long been recognised [40. The example in Fig. 7. show that there is both displacement of the atoms about the mean position of a layer but the layers themselves also undergo wave-like deformation. In order to quantify how much the layers bend during heating we carried out a number of MD simulations at various temperatures on systems containing 1008 atoms in 14 layers and examined the expansion in the interplane $c$ direction as well as the displacement of atoms in each layer from their mean position. The distribution about the mean turned out to be Gaussian with the stan- 
(a) Layer 2 initial
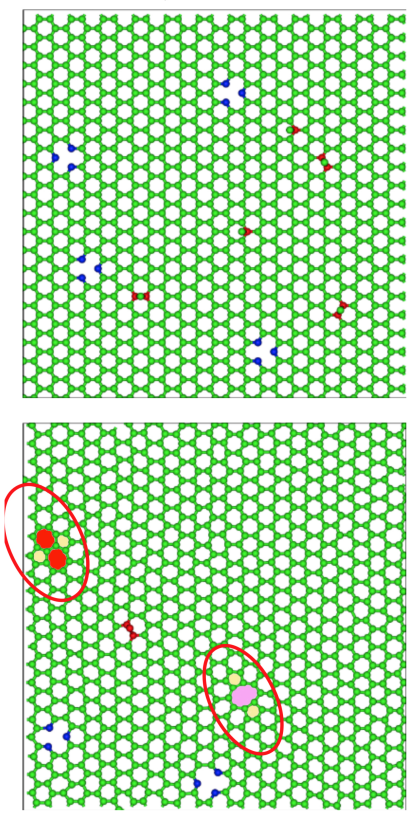

(b)

Layer 2 final
Layer 3 initial
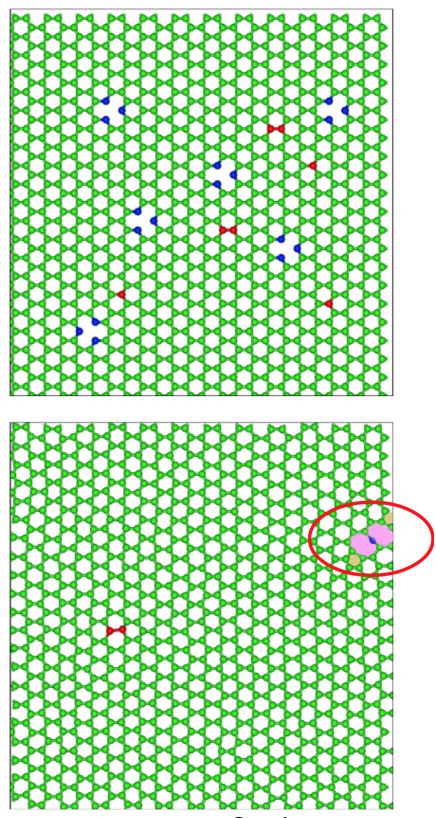

Layer 3 final

FIG. 16: Evolution of 10 vacancies and 5 spiro interstitials. (a) the assumed starting configuration; (b) the structure after annealing using MD for 2 ns at 2000 $\mathrm{K}$.

dard deviation being proportional to $T^{0.52}$ giving a value of $0.55 \AA$ at $2000 \mathrm{~K}$. We have not so far analysed this effect further but clearly this deformation is an important factor in facilitating defect motion.

Randomly placed interstitial atoms, that are close to

each other, combine, first to form chains, then ring structures and then mobile platelets between layers. As discussed in the literature over many years, this could help partially explain the lattice expansion in the $c$ direction due to the formation of additional layers in the system. The platelets are generally not bonded to adjacent layers but under co-ordinated edge atoms can bond and debond during high temperature simulations as the platelets move around between the layers.

Because the displacement of the atoms at high temperature is a combination of layer bending and displacement of the atoms about the mean position in the layer in the basal direction, a further study is required to quantify fully these effects.

\section{ACKNOWLEDGEMENTS}

This work was supported by United Kingdom EPSRC grant EP/M018822/1, UNIGRAF: Understanding and Improving Graphite for Nuclear Fission.

Kenny Jolley acknowledges funds from EDF to support a lectureship and a studentship 2016-2020. We thank Christopher Latham for useful discussions.

During the time this work was carried out our colleague at Loughborough, Malcolm Heggie, sadly passed away. Malcolm inspired us all with his knowledge of carbon and his enthusiasm and wit. We would therefore like to recognise the the contribution that Malcolm Heggie has made to us, this work and the carbon community in general by dedicating the paper to his memory.
[1] G. M. Laudone, C. M. Gribble, G. P. Matthews, Characterisation of the porous structure of gilsocarbon graphite using pycnometry, cyclic porosimetry and void-network modelling, Carbon 73 (2014) 61-70.

[2] B. März, J. Marrow, K. Jolley, Z. X. Zhou, R. Smith, M. Heggie, H. Wu, Mesoscopic structure features in synthetic graphite, Materials and Design 142 (2018) 268278.

[3] T. Lazauskas, S. Kenny, R. Smith, Influence of the prefactor to defect motion in alpha-iron during long time scale simulations, J. Phys. Condensed Matter 26 (2014) 395007.

[4] R. Smith, D. Bacorisen, B. Uberuaga, K. E. Sickafus, J. Ball, R. Grimes, Dynamical simulations of radiation damage in magnesium aluminate spinel, mgal2o4, J. Phys. Condensed Matter 17 (2005) 875-891.

[5] H. J. Christie, M. Robinson, D. L. Roach, D. K. Ross, I. Suarez-Martinez, N. A. Marks, Simulating radiation damage cascades in graphite, Carbon 81 (2015) 105-114.

[6] R. Nightingale, Nuclear Graphite, Academic Press, London (1962) 213.

[7] J. Tersoff, Empirical interatomic potential for carbon, with applications to amorphous-carbon, Physical Review Letters 61 (1988) 2879-2882.

[8] R. Smith, A classical dynamics study of carbon bombardment of graphite and diamond, Proc. Roy Soc. A 441 (1990) 143-155.

[9] A. Gras Marti, R. Smith, K. M. Beardmore, J. J. JiminezRodriguez, V. Konoplev, J. Fierron, Early stages of bump formation on ion-bombarded graphite, Computational Materials Science 3 (1995) 413-422.

[10] R. Smith, R. P. Webb, Energetic fullerene interactions with a graphite surface, Proc. Roy Soc. A 431 (1993) 495-499.

[11] R. Smith, K. Beardmore, Molecular dynamics studies of particle impacts with carbon-based materials, Thin Solid Films 272 (1996) 255-270.

[12] A. Chartier, L. Van Brutzel, J. Pageot, Irradiation damage in nuclear graphite at the atomic scale, Carbon 133 (2018) 224-231.

[13] T. Trevethan, M. I. Heggie, Molecular dynamics simulations of irradiation defects in graphite: Single crystal mechanical and thermal properties, Comp. Mater. Sci. 113 (2016) 60-65. 
[14] C. D. Latham, A. J. McKenna, T. P. Trevethan, M. I. Heggie, M. J. Rayson, P. R. Briddon, On the validity of empirical potentials for simulating radiation damage in graphite: a benchmark, J. Phys. Condensed Matter 27 (2015) 316301.

[15] D. Brenner, Empirical potential for hydrocarbons for use in simulating the chemical vapor-deposition of diamond films, Phys. Rev. B 42 (1990) 9458-9471.

[16] N. Marks, Modelling diamond like carbon with the environment dependent interaction potential, J. Phys. Condensed Matter 11 (2002) 2901-2927.

[17] P. Briddon, R. Jones, LDA calculations using a basis of Gaussian orbitals, Phys. Stat. Solidi B 217 (2000) 131171.

[18] T. P. Senftle, S. Hong, M. M. Islam, S. B. Kylasa, Y. Zheng, Y. K. Shin, C. Junkermeier, R. Engel-Herbert, M. J. Janik, H. M. Aktulga, T. Verstraelen, A. Grama, A. C. T. van Duin, The ReaxFF reactive force-field: development, applications and future directions, NPJ Computational Materials 2 (2016) 15011.

[19] R. Smith, K. Jolley, C. Latham, M. Heggie, A. van Duin, D. van Duin, H. Wu, A reaxff carbon potential for radiation damage studies, Nuclear Instruments and Methods in Physics Research Section B: Beam Interactions with Materials and Atoms 393 (2017) 49-53.

[20] L. Li, S. Reich, J. Robertson, Structural defects in graphene, Phys. Rev. B 72 (2005) 184109.

[21] F. Banhart, J. Kotakoski, A. V. Krasheninnikov, Defect energies of graphite, ACSNano 5 (2011) 26-41.

[22] A. Gulans, A. Krasheninnikov, M. Puska, R. Nieminen, Bound and free self-interstitial defects in graphite and bilayer graphene: A computational study, Phys. Rev. B 84 (2011) 024114.

[23] G. Lee, C. Wang, E. Yoon, N. Hwang, K. Ho, Vacancy defects and the formation of local haeckelite structures in graphene from tight-binding molecular dynamics, Phys. Rev. B 74 (2006) 245411.

[24] L. Xu, G. Henkelman, Adaptive kinetic monte carlo for first-principles accelerated dynamics, J. Chem.Phys. 129 (11) (2008) 114104.

[25] C. Scott, S. Blackwell, L. Vernon, S. Kenny, J. M. Walls, R. Smith, Atomistic surface erosion and thin film growth modelled over realistic time scales, Journal of Chemical Physics 135 (17) (2011) 174706.

[26] B. P. Uberuaga, R. Smith, A. R. Cleave, G. Henkelman, R. W. Grimes, A. F. Voter, K. E. Sickafus, Dynamical simulations of radiation damage and defect mobility in mgo, Phys. Rev. B 71 (2005) 104102.

[27] T. Trevethan, C. Latham, M. I. Heggie, M. J. Rayson, P. R. Briddon, Interlayer vacancy diffusion and coalescence in graphite, Phys. Rev. B 90 (2014) 174108.

[28] M. Heggie, I. Suarez-Martinez, C. Davidson, G. Haffenden, Buckle, ruck and tuck: A proposed new model for the response of graphite to neutron irradiation, J. Nucl. Mater. 413 (2011) 150-155.

[29] A. Asthana, Y. Matsui, M. Yasuda, K. Kimoto, T. Iwatac, K. Ohshimaa, Investigations on the structural disordering of neutron-irradiated highly oriented pyrolytic graphite by x-ray diffraction and electron microscopy, J. Applied. Crystallography 38 (2005) 361-367.

[30] J. F. Ziegler, U. Littmack, J. P. Biersack, The stopping and range of ions in solids, in: Ion Implantation Science and Technology (Second Edition), Elsevier, 1988, pp. 361.

[31] S. Plimpton, Fast parallel algorithms for short-range molecular dynamics, Journal of computational physics 117 (1) (1995) 1-19.

[32] G. Henkelman, B. P. Uberuaga, H. Jónsson, A climbing image nudged elastic band method for finding saddle points and minimum energy paths, The Journal of chemical physics 113 (22) (2000) 9901-9904.

[33] D. Perez, E. Cubuck, A. Waterland, E. Kaxiras, A. Voter, Long-time dynamics through parallel trajectory splicing, J. Chem. Theory Comput. 12 (1) (2016) 18-28.

[34] A. El-Barbary, R. Telling, C. Ewels, M. I. Heggie, P. R. Briddon, Structure and energetics of the vacancy in graphite, Phys. Rev. B 68 (2003) 144107.

[35] C. Latham, M. Heggie, J. Gamez, I. Suarez-Martinez, C. P. Ewels, P. R. Briddon, The di-interstitial in graphite, J. Phys. Condensed Matter 20 (2008) 395007.

[36] G. J. Dienes, Mechanism for self-diffusion in graphite, J. Appl. Phys. 23 (1952) 1194-1200.

[37] M. Monthioux, J. Charlier, Giving credit where credit is due: The stone-(thrower)-wales designation revisited, Carbon 75 (2014) 1-4.

[38] P. Thrower, R. Mayer, Point defects and self-diffusion in graphite, Phys. Stat. Sol. A. 47 (1978) 11-37.

[39] A. Stone, D. Wales, Theoretical studies of icosahedral c60 and some related species, Chem. Phys. Lett. 128 (1986) 501-503.

[40] R. Smith, R. P. Webb, Energetic fullerene interactions with a graphite surface, Proc. Roy. Soc. 441 (1993) 495499. 